\title{
An Interband Tunnel Oscillator: Intrinsic Bistability and Hysteresis of Trapped Hole Charge in a Double-Barrier Structure
}

\author{
F. A. BUOT
}

Naval Research Laboratory, Washington, D. C. 20375-5320

\begin{abstract}
We introduced a novel high-frequency source based on interband tunneling. A polarization-induced oscillation of trapped-hole-charge occurs in an AlGaSb/InAs/ $\mathrm{AlGaSb}$ resonant tunneling device. Rate equations for Zener tunneling, polarization, and electron-hole recombination is used to analyze the nonlinear dynamics of this device structure. The nonoscillatory state is unstable against the limit-cycle operation. The amplitude of trapped hole oscillation increases with bias, but the time-averaged values can be approximated by a step function. These lead to the hysteresis of the averaged trapped hole charge in $\mathrm{AlGaSb}$ barrier, and to the experimental intrinsic bistability in $\mathrm{AlGaSb} / \mathrm{InAs} / \mathrm{AlGaSb}$ resonant tunneling device. Large-scale time-dependent simulation of quantum transport with interband-tunneling dynamics is needed for the design optimization of this novel class of oscillator useful for high-bandwidth applications.
\end{abstract}

Keywords: Limit cycle, hysteresis, trapped holes, intrinsic bistability, zener effect, resonant tunneling devices, self-oscillation

\section{INTRODUCTION}

The 'hetero' junction has become the basic building block of most of the advanced high-speed devices for electronic, microwave, and optoelectronic applications [1,2]. Moreover, tunneling devices exhibit autonomous oscillation, similar to Gunn effect devices but at much higher frequencies in nanometric sizes. For conventional resonant tunneling devices (RTD) [3-6], this occurs when the device is operating in the negative-differentialresistance (NDR) region, just after the resonant current peak. The oscillation addressed in this paper occurs before the resonant current peak, based on interband tunneling in RTD with staggered band-gap alignment.

A staggered band-edge alignment can be realized by using InA/AlSb hetero junctions, Figure 1a. In a simple implementation of a novel interband tunnel high-frequency source, a deeper quantum-well-for-holes is desirable which can support a localized hole state; this is obtained by using In $\mathrm{As} / \mathrm{AlGaSb}$ heterojunctions, Figure 1b. Unless otherwise specified, quantum well refers to the conduction band edge and conduction-band electrons. 

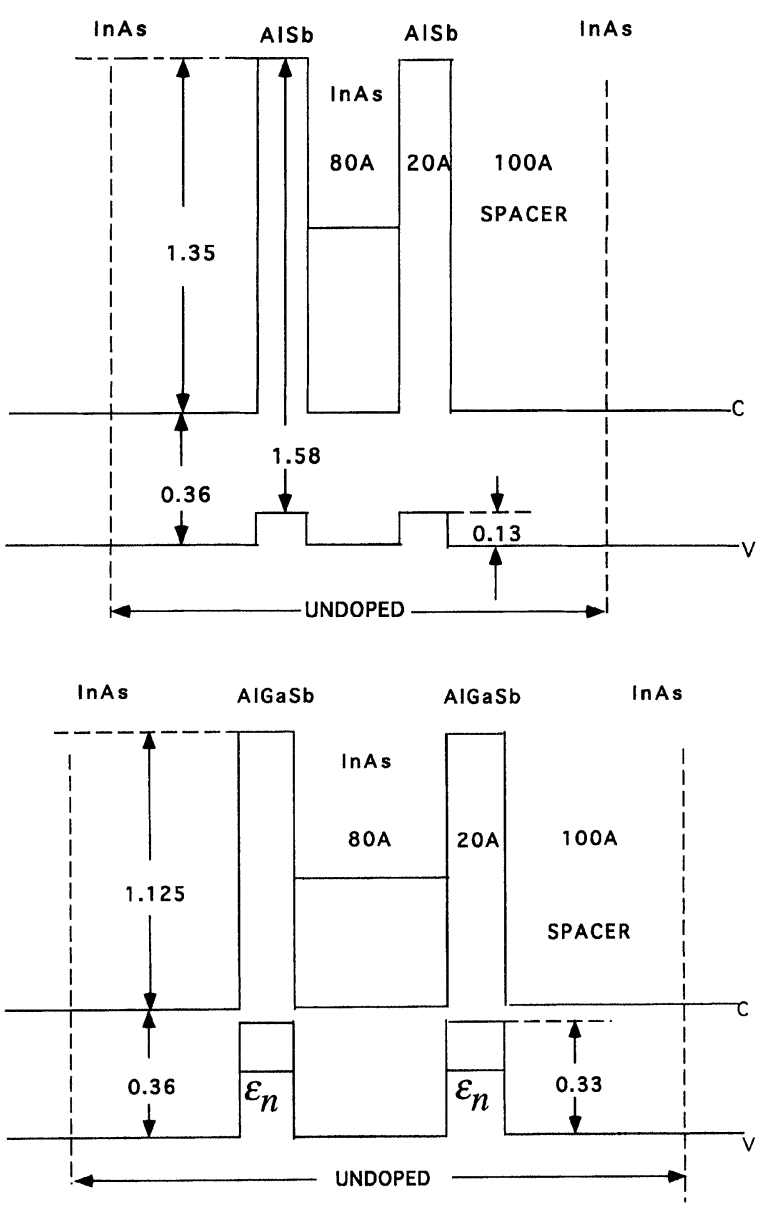

FIGURE 1 (a) Energy band edge (EBE) alignment of RTD using InAs/AlSb heterojunction. (b) Energy band edge alignment of RTD using InAs/AlGaSb heterojunction. Approximated band-edge offsets are indicated in electron volts.

The new mechanism of modulating the resonant energy level in the quantum well with respect to the energy distribution of the electrons from the emitter can simply be described through the oscillatory build-up and decay of the polarization pairing between electrons in the quantum well and trapped holes in the barrier. This modulation is controlled by trapped holes (similar to base charges of a bipolar transistor). Thus, for the first time we realize an autonomous control of a significant current by an interband process.

We refer to the polarization pair as a duon, since this Coulomb-correlated $\mathrm{e}-\mathrm{h}$ pair only moves in the transverse direction, in contrast to exciton. This is in analogy to the use of trion in referring to a correlated exciton and electron in adjacent quantum-well heterostructure [11]. In Sec. 3, we introduce the physics of the duon dynamics. The limit cycle solution leads to an oscillatory voltage drop between the quantum well and the barrier. Since common experimental techniques are incapable of investigating these oscillations $[3,4,8]$, the current-voltage $(I-V)$ characteristic is also calculated in Sec. 4. The results agree with the experiment. In Sec. 5, we draw some conclusions, as well as give a summary of this paper.

\section{HIGH-FREQUENCY OPERATION}

Under bias, when the localized electrons in the $\mathrm{AlGaSb}$ barrier of Figure $1 \mathrm{~b}$ see the available states these electrons tunnel to the drain by Zener transition, leaving behind holes in a discrete 'longitudinal' energy level, $\varepsilon_{n}$. This is initiated when a matching of $\varepsilon_{n}$ with available conductionband states in the drain first occurs, at $k_{z}^{D^{2}} \approx k_{F}^{D^{2}}$ in Figure 2. Figure 2 serves to define several quantities used in the calculations of Sec. 4. The drain acts as a sink due to unoccupied states above $k_{F}^{D}$ that could satisfy the conservation of transverse crystal momentum associated with $\varepsilon_{n}$.

As hole charging occurs, Figure 3 (1), the polarization Figure 3 (2) creates a high-field domain, at the expense of the potential drop between the contact and the barrier. When the situation shown in Figure 3 (3) is reached, the onset of other mechanisms for hole discharging may also occur, namely, thermal activation of the valence electrons in the continuum to recombine with localized holes, or loss of any bound hole states in the barrier. The 'hole leakage' will restore the large voltage drop between the barrier edge and the right contact. The situation shown in Figure 3 (1) is revisited, after which the process repeats. Therefore, oscillations of the hole charging of the $\mathrm{AlGaSb}$ barrier can occur at high frequency by virtue of the nanometric features of 


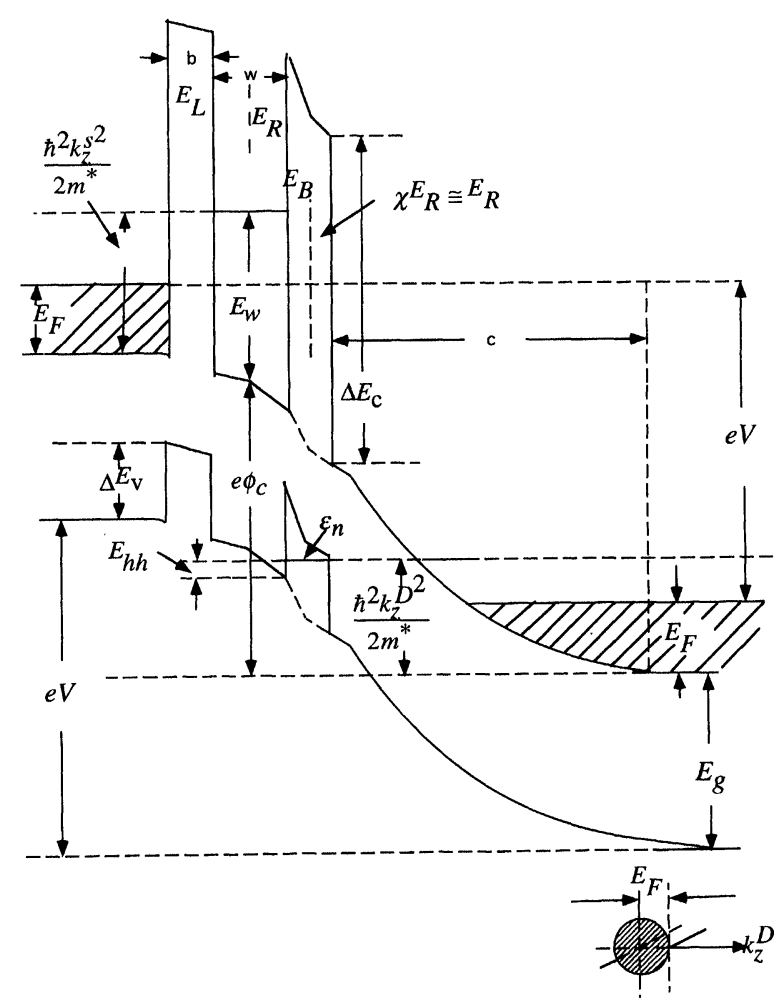

FIGURE 2 Schematic averaged EBE profile showing the various quantities used in the calculations of $I-V$ plot. The shaded regions in the lower right-hand corner indicate the occupied transverse and longitudinal momentum states in the drain.

the device. We estimate the charging time, which is the dominant time scale of the problem, to be about 200-1000 femtoseconds for a heavy-hole state in $\mathrm{AlGaSb}$ with indirect-gap interband tunneling through Keldysh effect.

The above dynamical process limits the amount of hole charge that can be trapped in the barrier as a function of bias. The interband recombination process can not compete with the conduction-band electron tunneling process through the barrier [3]. The criteria for either detailed balance or oscillatory behavior are governed by the two characteristic times, namely, the polarization-charge buildup time, $\tau_{B}$, and the charge-leakage time, $\tau_{L}$. If $\tau_{B}>\tau_{L}$, then the charging process will be lagging behind the discharging process and oscillations

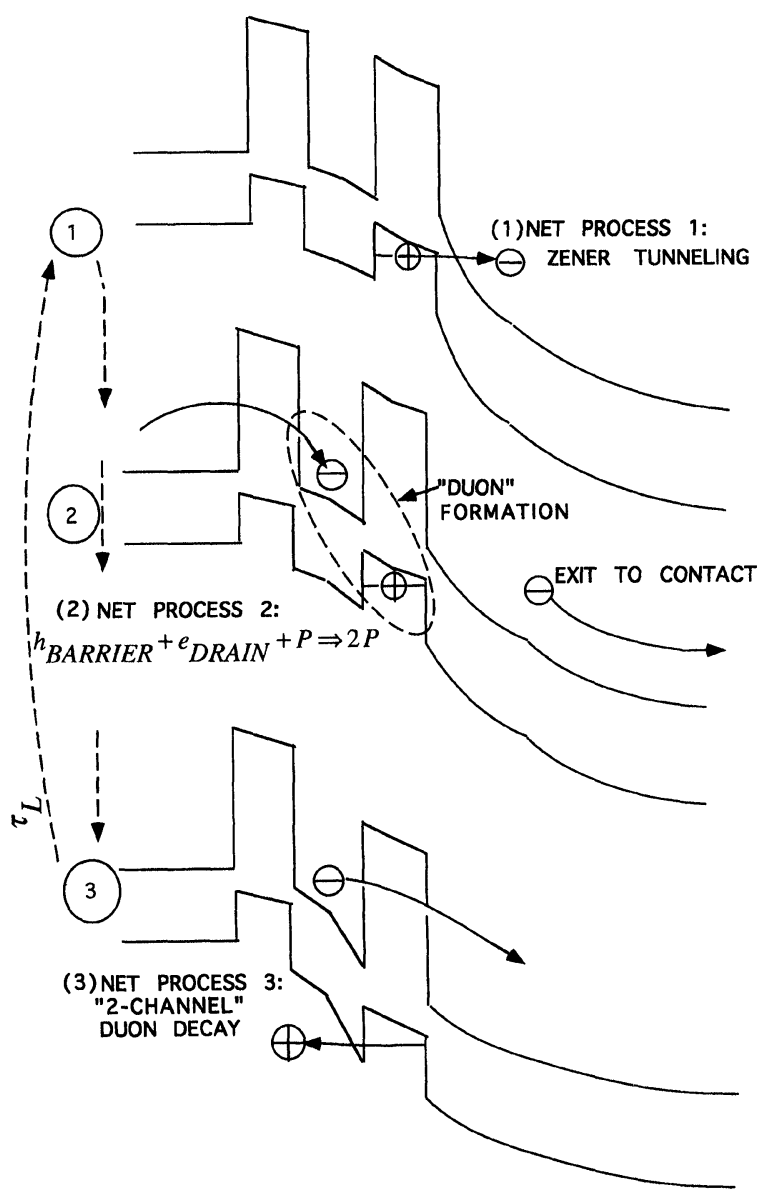

FIGURE 3 (1) $\mathrm{e}-\mathrm{h}$ generation by Zener tunneling. (2) duon generation is through an autocatalytic process. (3) three mechanisms for hole discharging are mentioned in the text.

will result. This holds true in conventional RTD and single-electron devices [10]. It is here estimated that $\tau_{B}>\tau_{L}$, by virtue of several possible fast holedischarge channels mentioned above. The 'bookeeping equations' ('chemical kinetics' modeling) of the device operation follows.

\section{POLARIZATION PAIRING DYNAMICS}

We derive here the coupled rate equations for the processes in Figure 3. The duon formation is via the polarization-induced transport of conduction electrons from the emitter to the quantum well, 
coupled with a succeeding $\mathrm{e}-\mathrm{h}$ generation by Zener tunneling. In process (2), the Zener-tunneled electron flows to the metallic contact of the drain, and this is substituted by the tunneled conduction electron from the emitter to form a duon. We observe here an 'autocatalytic' or positive-feedback aspect of the duon formation.

Let $G$ be the maximum rate of $\mathrm{e}-\mathrm{h}$ generation by Zener tunneling. Note that $G$ is a direct measure of the applied bias. The duon generation rate with 'three interacting components' can be expressed as $\tilde{\Delta} \mathscr{N}_{B}^{2} P$, where $\mathscr{N}_{B}$ is the concentration of 'unpaired' holes which is equal to the concentration of 'exiting' electrons produced by Zener tunneling, $P$ is the concentration of duons, and $\tilde{\Delta}$ is a parameter which is expected to acquire, in appropriate ranges, a nonlinear dependence on $P$ as discussed below. We can now write the 'effective' generation rate of unpaired trapped holes in the barrier as

$$
\frac{\partial}{\partial t} \mathscr{N}_{B}=G-\tilde{\Delta} \mathscr{N}_{B}^{2} P
$$

The total concentration of trapped holes in the barrier, $Q_{B}$, at any time is given by $Q_{B}=\mathcal{N}_{B}+P$.

For large $P$ the transfer of conduction electrons from the emitter to the quantum well becomes more efficient since it is approaching the resonance peak and $\tilde{\Delta}$ increases. For very small $P$, the concentration of resident electrons already existing in the quantum well (refer to Fig. 4) will also render the polarization pairing to be much more efficient, and hence a larger $\tilde{\Delta}$, than for the intervening ranges between small $P$ and large $P$. This acquired nonlinearity of the parameter $\tilde{\Delta}$ is important in establishing a limit cycle operation of the device, since it limits the growth of the solution from the unstable focus. Indeed, we shall see that the 'linear' criterion for unstable stationary operation is that $F(G)>4 \tilde{\Delta}$ where $F(G)$ is a constant for a fixed bias.

The decay rate for $P$ is expected to saturate for very large $P$. In all foreseeable cases, we may express the decay rate of the duon concentration as

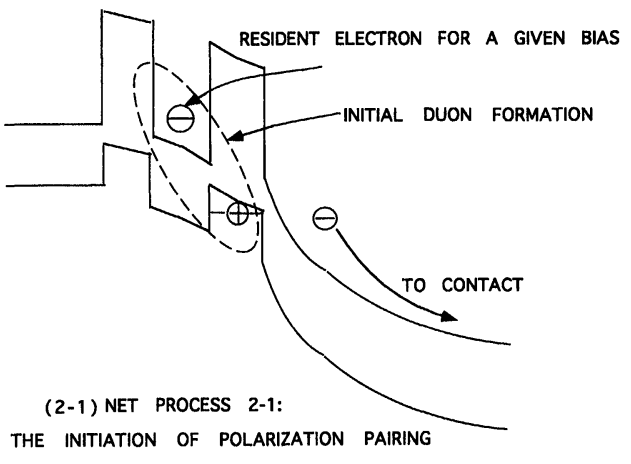

FIGURE 4 More efficient generation of initial polarization pairs or duons is due to the initial resident excess electron in the quantum well for a given applied bias.

$=\alpha P /(1+\beta P)$, where $1 / \beta$ represents the sum of available states in the valence band of the quantum well region and the states participating in thermal recombination; otherwise it represents the actual concentration of hole states in the barrier in the case of the loss of bound hole state. This is similar to the decay law ubiquitous in chemical kinetics [12]. The parameter $\alpha$ is the decay rate constant and $\alpha / \beta$ is the value of the saturated decay rate of duons. Therefore, we can now write the duon generation rate as

$$
\frac{\partial}{\partial t} P=\tilde{\Delta} \mathscr{N}_{B}^{2} P-\frac{\alpha P}{1+\beta P}
$$

As seen in Eq. (4) below, the physical situation corresponds to $\alpha / \beta>G$. Indeed, we can estimate that $1 / \tau_{B} \approx G \approx G$ and $1 / \tau_{L} \approx \alpha / \beta$. Therefore $\alpha / \beta>G$ implies that $\tau_{B}>\tau_{L}$.

\subsection{Stability Analysis}

The stationary solution for a fixed bias (implying a fixed $G$ ) to Eqs. (1) and (2) is given by

$$
G=\tilde{\Delta} \mathscr{N}_{B}^{2} P=\frac{\alpha P}{1+\beta P}
$$


The total stationary trapped hole concentration, $Q_{B}$, is thus given by

$$
Q_{B}=\mathscr{N}_{B}^{0}+P^{0}=\frac{G}{\alpha-\beta G}+\left(\frac{\alpha-\beta G}{\tilde{\Delta}}\right)^{1 / 2},
$$

which is a sum of an increasing and a decreasing function of bias. The more accurate average value under a limit cycle oscillation is shown in Sec. 3.2 to be approximately independent of bias. Since $Q_{B}$ and $P^{0}$ are constants, the duon production rate is via the transfer of conduction electrons from the emitter to the quantum well and the duon decay rate is via transfer of conduction electrons from the quantum well to the drain. Thus, the d.c. operation no longer involves interband processes, as schematically shown in Figure 5.

Complete analysis of the stability of the stationary solution as well as the full derivation of the limit cycle solution will be given elsewhere. By transforming to dimensionless variables: $\Pi=$ $\beta P, \quad \mathscr{Q}=\beta \mathcal{N}_{B}, \quad \Delta=\tilde{\Delta}(1 / \beta)^{2} / \alpha, \quad \mathscr{G}=G /(\alpha / \beta)$ $<1.0$, the criterion for unstable stationary solution is that $(1-\mathscr{G})^{3}>4 \Delta$. Following a perturbation technique using multiple time scales [13,14], we have obtained to second order in the smallness parameter, $\varepsilon=\left(\left\{\Delta-\Delta_{c}\right\} / \Delta_{2}\right)^{1 / 2}$, where $\Delta_{c}=$ $\left((1-\mathscr{G})^{3} / 4\right)$ and $\Delta_{2}$ comes from the expansion of $\Delta$ near $\Delta_{c}$ in powers of $\varepsilon$, the limit cycle solution. This is given as

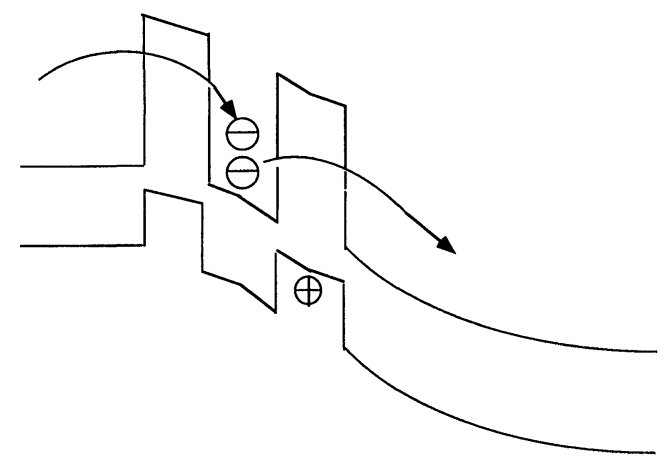

FIGURE 5 At steady state the two conduction-electronmediated duon decay and generation processes are balanced resulting in steady-state current across the double barrier structure.

$$
\left(\begin{array}{c}
\Pi \\
\mathscr{Q}
\end{array}\right)=\left(\begin{array}{c}
\Pi^{0} \\
\mathscr{2}^{0}
\end{array}\right)+\varepsilon\left(\begin{array}{c}
p_{0} \\
q_{0}
\end{array}\right)+\varepsilon^{2}\left(\begin{array}{c}
p_{1} \\
q_{1}
\end{array}\right)+O\left(\varepsilon^{3}\right),
$$

The two column vectors $\left(\begin{array}{l}P_{0} \\ q_{0}\end{array}\right)$ and $\left(\begin{array}{l}P_{1} \\ q_{1}\end{array}\right)$ contain the oscillating factors associated with first and second orders, respectively. We note that $\left(\begin{array}{l}P_{1} \\ q_{1}\end{array}\right)$ and higherorder terms also contain higher-order time-independent terms which increases with bias. Therefore, the average value of $\left(\begin{array}{l}\pi \\ 2\end{array}\right)$ is given by

$$
\left(\begin{array}{l}
\pi \\
\mathscr{Q}
\end{array}\right)_{\text {average }}=\left(\begin{array}{l}
\pi^{0} \\
\mathscr{2}^{0}
\end{array}\right)+\text { higher-order corrections, }
$$

where the leading higher-order corrections comes from these time-independent terms.

\subsection{Dependence with Bias}

Based on a finite limit of the amplitude (which only depends on the slow time scale), the limit cycle solution is found to occur within the range of values of the parameter $\Delta$ where the 'linear' criterion for unstable focus $(1-\mathscr{G})^{3}>4 \Delta$ still holds. This is analogous to the numerically simulated limit cycle of AlGaAs/GaAs/AlGaAs double-barrier heterostructure operating in the NDR region [7]. For values of $\Delta$ near the critical point, the rate of change with respect to $\mathscr{G}$ is approximately zero. Indeed, we have from Eq. (4),

$$
\frac{d \beta Q_{B}(\mathscr{G})}{d \mathscr{G}}=\frac{1}{(1-\mathscr{G})^{2}}-\left(\frac{1}{4 \Delta(1-\mathscr{G})}\right)^{1 / 2} \leq 0
$$

where the equality is obtained at $\Delta=\Delta_{c}=$ $(1-\mathscr{G})^{3} / 4$. Since $\mathscr{G}$ is our measure of the applied voltage applied, we conclude from Eq. (7) and by taking into account the higher-order correction terms which increases with $\mathscr{G}$ that the average total hole charge trapped in the barrier is approximately independent of bias. 
Denoting the leading time-dependent part of $Q_{B}(t)$ as $\delta Q_{B}(t)$, the total trapped hole charge in the barrier oscillates with amplitude that increases with bias and is given by

$$
\begin{aligned}
\delta Q_{B}(t) & =\frac{4\left(\left|\Delta-\Delta_{c}\right|\right)^{1 / 2}}{\beta} \\
& {\left[\frac{\mathscr{G}}{(1-\mathscr{G})^{4}\left|\left\{-8-17 \mathscr{G}+36 \mathscr{G}^{2}-11 \mathscr{G}^{3}\right\}\right|}\right]^{1 / 2} } \\
& {[\cos \Omega t-\sin (\Omega t+\Phi)] }
\end{aligned}
$$

where the frequency $\Omega$ consists of a function of $\mathscr{G}$ plus higher-order terms, and $\Phi=\{\mathscr{G} /(1-\mathscr{G})\}^{1 / 2}$ $>0$. The oscillation amplitude grows in response to the increasing maximum electric field in the depletion region with the applied bias, since the maximum $\mathrm{e}-\mathrm{h}$ generation rate, $\mathscr{G}$, by Zener tunneling [15] increases with bias.

\section{INTRINSIC BISTABILITY IN InAs $/ \mathbf{A l}_{x} \mathbf{G a}_{1-x} \mathbf{S b}$ RTD}

The time-averaged hole charge in the barrier is referred to as $Q(\mathrm{AlGaSb})=e Q_{B}$, where $e$ is the positive unit charge. This value is approximately independent of bias in Sec. 3, after an abrupt increase at $k_{z}^{D^{2}} \approx k_{F}^{D^{2}}$ in Figure 2. The selfconsistency of the potential alone, in Figure 2, demands that the polarization and hence $Q(\mathrm{Al}-$ $\mathrm{GaSb}$ ) increases monotonically with bias. We shall see that the simultaneous solution to these two requirements, plus the continuity condition, leads to a 'parallelepiped' hysteresis of the trapped hole charge in the barrier.

We only need three field parameters to include a concave EBE profile in the barrier region. The inflection point is assumed to have a measure zero as far as the integration of the fields to obtain the total voltage drop across the device is concerned. For nonzero average value of $\mathscr{N}_{B}$, which is the concentration of "unpaired" trapped holes, we àlso expect a nonzero superposed polarization between the barrier and spacer layer to be affecting the potential profile, as indicated by a simple 'kink' in the spacer region of Figure 2. We estimate the fourth field parameter in the second half of the barrier as proportional to $E_{R}$, as the figure suggests with proportionality factor, $\chi(V) \leq 1.0$, and still maintain the physical requirement of concave EBE profile in this region. This accounts for nonzero average $\mathcal{N}_{B}$.

From Figure 2, the trapped hole charge in the second barrier is given by the expression: $\chi E_{R}-$ $E_{B}=Q(\mathrm{AlGaSb}) / \varepsilon$, from the Poisson equation. We estimate the proportionality factor $\chi$ is close to unity and positive. From the requirement of faster voltage drop in the barrier region in Figure 2, we must have $E_{B}$ more negative than $\chi E_{R}$, thus we obtain $Q(\mathrm{AlGaSb})>0$ consistent with the trapped hole charge in the second barrier.

\subsection{Hysteresis of Trapped Hole Charge}

The positive applied bias, $V$, in Figure 2 , is given by the following expression, $e V=e\left|E_{L}\right|(b+w / 2)$ $+e\left|E_{R}\right|(w / 2)+E_{g}+\left(\hbar^{2} k_{z}^{D^{2}} / 2 m^{*}\right)-E_{h h}$, where $w$ and $b$ are the width of the quantum well and barrier, respectively. We use the Poisson equation to eliminate $\left|E_{R}\right|$ in terms of $\left|E_{L}\right|$. Since all fields on the average have negative sign for positive applied voltage, we may also write Poisson equation as $\left|E_{R}\right|-\left|E_{L}\right|=\left|Q_{w}\right| / \varepsilon,\left|E_{B}\right|-\chi\left|E_{R}\right|=Q(\mathrm{AlGa} \mathrm{Sb}) / \varepsilon$. Therefore, we obtain the following expression for the trapped hole charge,

$$
\begin{aligned}
Q(\mathrm{AlGaSb})= & \frac{2 \varepsilon \chi}{e w}\left[\left(E_{g}-E_{h h}\right)\right. \\
& -\left(e V-e\left|E_{L}\right|\left(b+\frac{w}{2}\right)-e\left|E_{B}\right| \frac{w}{2 \chi}\right) \\
& \left.+\frac{\hbar^{2} k_{z}^{D^{2}}}{2 m^{*}}\right] .
\end{aligned}
$$

The trapped hole charge is an increasing function of $k_{z}^{D^{2}}$ in Eq. (9), since the 'polarization', 
without the constraint of quantum transport nonlinear dynamics, should increase monotonically with bias.

The independence with bias beyond a threshold value of the trapped holes in Sec. 3 is expressed here by a step function

$$
Q(\mathrm{AlGaSb})=Q_{h} \Theta\left(k_{z}^{D^{2}}-k_{F}^{D^{2}}\right)
$$

The simultaneous solutions of Eqs. (9) and (10) is shown graphically in Figure 6, Eq. (9) for $Q(\mathrm{AlGaSb})$ vs. $k_{z}^{D^{2}}$ is approximated by positive sloping lines. Upon applying the continuity condition, open circles and solid circles are solutions for the increasing voltage sweep and decreasing voltage sweep, respectively. A 'parallelepiped' hysteresis of trapped hole charge as a function of bias is clearly indicated.

\subsection{Hysteresis in the $I-V$ Characteristics}

This is obtained by describing the whole length of the device by three independent fields, namely, $E_{L}$, $E_{R}^{\prime}$ and $E_{B}^{\prime}$. Note that the field $\chi E_{R}$ used before is only valid in the right-half of the barrier region, by virtue of nonzero average concentration of 'unpaired' trapped holes, $\mathcal{N}_{B}$, as indicated in Figure 2. The field $E_{L}$ is as defined before, whereas $E_{R}^{\prime}$ is defined by the relation: $E_{R}^{\prime}(b / 2+w / 2)=$ $E_{R}(w / 2)+E_{B}(b / 2)$, and $E_{B}^{\prime}$ is the constant field approximation for the rest of the device of dimension $[(b / 2)+c]$. As a consequence, we also have the following relation: $\left|E_{R}^{\prime}\right|-\left|E_{L}\right|=\alpha^{\prime}\left|Q_{w}\right| / \varepsilon$

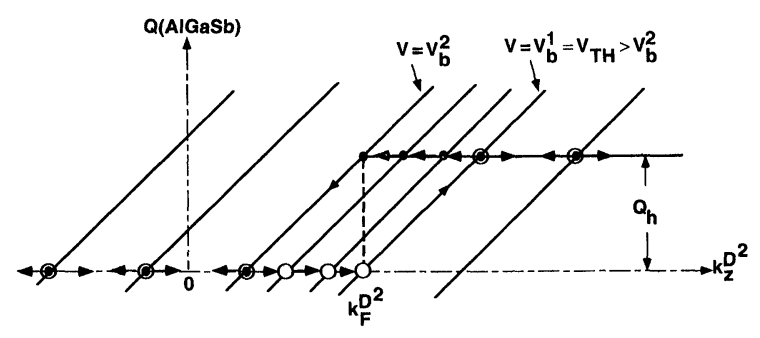

FIGURE 6 Graphical solution of Eqs. (9) and (10). and $\left|E_{R}^{\prime}\right|-\left|E_{B}^{\prime}\right|=\beta^{\prime} \mid Q(\mathrm{AlGaSb}) / \varepsilon$, where $\alpha^{\prime}$ and $\beta^{\prime}$ proportionality constants. Thus we may write $e V=e\left|E_{L}\right|(b+w / 2)+e\left|E_{R}^{\prime}\right|(w+b) / 2+e\left|E_{B}^{\prime}\right|(b / 2+c)$. We then expressed $\left|E_{R}^{\prime}\right|$ and $\left|E_{B}^{\prime}\right|$ in terms of $\left|Q_{w}\right|$, $Q(\mathrm{AlGaSb})$, and $\left|E_{R}\right|$. We also use the relation: $e\left|E^{L}\right|=e\left(V-\phi_{c}\right) /(2 b+w)$, where $e \phi_{c}=e V-$ $E_{w}+\hbar^{2} k_{z}^{s^{2}} / 2 m^{*}$ to obtain the result

$$
\begin{aligned}
\left|Q_{w}\right|= & \frac{2 \varepsilon}{\alpha^{\prime}\left((b+c)+\frac{w}{2}\right)} \\
& \left\{\frac{\hbar^{2} k_{z}^{s^{2}}}{2 m^{*}}-E_{w}+\frac{V}{2 \xi}+\frac{\beta^{\prime} Q(\mathrm{AlGaSb})}{2 \xi\left(\frac{\varepsilon}{b / 2+c}\right)}\right\}
\end{aligned}
$$

where we have $\xi=(2 b+w+c) /(2 b+w)$. The quantum transport requirement for $Q_{w}$ was given by Buot and Rajagopal $[9,10]$ as

$$
\begin{aligned}
\left|Q_{w}\right|= & \frac{e m^{*}}{\pi \hbar^{2} \beta} \ln \left\{1+\exp \beta\left(E_{F}-\frac{\hbar^{2} k_{z}^{s^{2}}}{2 m^{*}}\right)\right\} \\
& \left(\frac{\tau_{d}}{\tau_{e}}\right) \Theta\left(k_{z}^{2}\right),
\end{aligned}
$$

which is zero for $k_{z}^{s^{2}} \leq 0,1 / \tau_{d}=1 / \tau_{e}+1 / \tau_{c}$, where $1 / \tau_{c}$ is the effective rate of decay of $Q_{w}$ into unoccupied collector states and $1 / \tau_{e}$ is equal to the rate of supply of electrons from the emitter to the quantum well. The simultaneous solution of Eqs. (11) and (12) is also graphically obtained as shown in Figure 7. In Figure 7a, Eq. (11) is approximated by parallel sloping lines. The values for $Q(\mathrm{Al}-$ $\mathrm{GaSb}$ ) are solutions obtained from Figure 6 which create an offset in the sloping lines of Eq. (11), leading to higher values of $Q_{w}$ as indicated by the dotted arrows. For the increasing voltage sweep, the solutions for $Q_{w}$ are given by the intersection points $S_{1}, S_{2}, S_{3}^{F}, S_{4}^{F}, S_{5}^{F}, S_{6}^{F}$ (low), $S_{6}$ (high), $S_{7}$ and $S_{8}$. For the reverse voltage sweep, the corresponding solution points are $S_{8}, S_{7}, S_{6}, S_{5}^{R}, S_{4}^{R}, S_{3}^{R}, S_{2}^{R}$ [high], $S_{2}$ [low], and back to $S_{1}$.

The RTD current can be approximated by $Q_{w} /$ $\tau_{c}$. The resulting $I-V$ has all the salient features of 

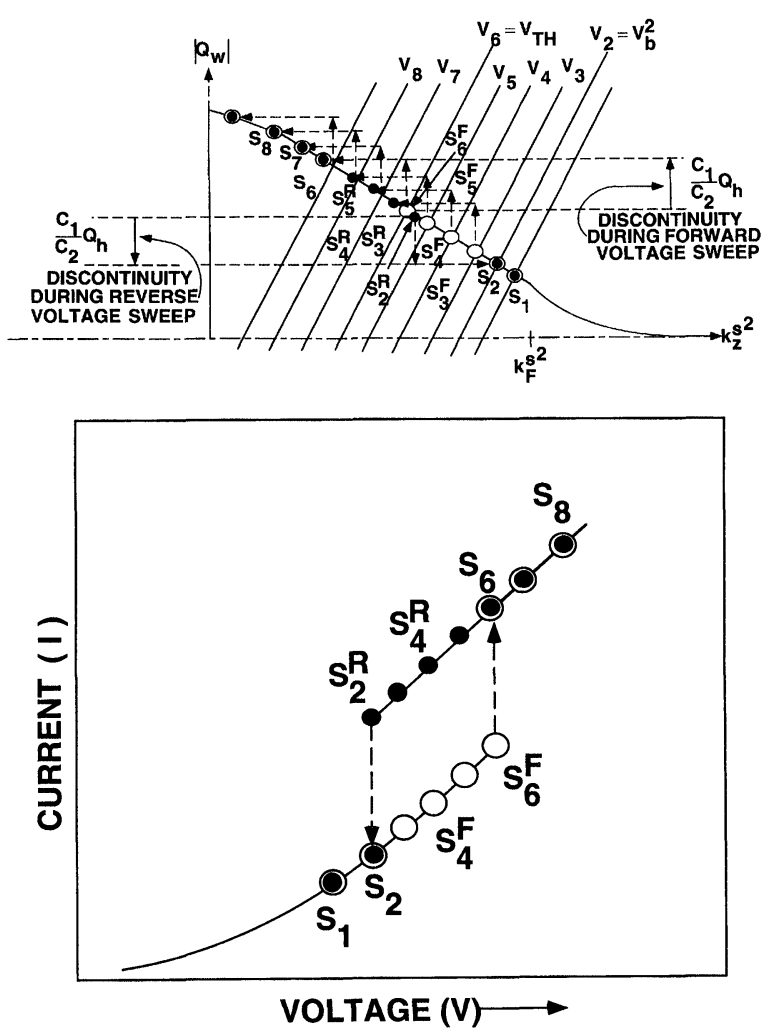

FIGURE 7 (a) Graphical solution of Eqs. (11) and (12). (b) The solution for the $I-V$ characteristic showing 'parallelepiped' hysteresis occurring before the RTD current peak, in agreement with the experiment.

the experimental results [9], shown in Figure 7b with corresponding solution points indicated. This result serves as indirect experimental evidence on the ability of this device to function as a highfrequency source.

\section{CONCLUSIONS}

The nonlinear dynamics of coupled systems of duons and 'unpaired' trapped-holes in RTD with staggered band-gap alignment provides a fundamental physical basis of the experimental intrinsic bistability of $\mathrm{AlGaSb} / \mathrm{InAs} / \mathrm{AlGaSb}$ RTD. Zener tunneling, stimulated formation of duons, and the self-oscillation of the trapped hole charge provide the mechanism behind a new high-frequency source introduced in this paper. We note that the RTD current peak occurs when the quantum-well energy level aligns with the bottom of the conduction band of the emitter. In some emitter designs, the Fermi level adjacent to the barrier increases as this alignment is approached, leading to a high-transconductance with the high-field domain acting as a self-gate. Thus, the transconductance of this 'self-gated'-transistor oscillator can be made large, yielding a novel high-frequency source with a usable power.

The 'chemical kinetics' modeling used above to analyze the device operation adds to the store of analytical tools for characterizing complex devices. However, large-scale time-dependent simulation is needed for further research and design optimization. Besides potential applications in communications, defect engineering would also make it as a triggering element in semiconductor lasers.

\section{Acknowledgements}

The author is thankful to Dr. A. K. Rajagopal for helpful comments on the manuscript. He gratefully acknowledge partial support from the Office of Naval Research.

\section{References}

[1] Yu, E. T., McCaldin, J. O. and McGill, T. C. (1992). In Solid-State Physics, Advances in Research and Applications, San Diego: Academic Press.

[2] Ting, D. Z. Y., Yu, E. T. and McGill, T. C. (1992). "Multiband treatment of quantum transport in interband tunnel devices", Phys. Rev., B45, 3583-3592.

[3] Jensen, K. L. and Buot, F. A. (1991). "Numerical simulation of intrinsic bistability and high-frequency current oscillations in resonant tunneling structures", Phys. Rev. Lett., 66, 1078-108.

[4] Biegel, B. A. and Plummer, J. D. (1996). "Comparison of self-consistency iteration options for Wigner function method of quantum device simulation", Phys. Rev., B54, $8070-8082$.

[5] Buot, F. A. and Rajagopal, A. K. (1993). "High-frequency behavior of quantum-based devices: equivalent circuit, 
nonperturbative response, and phase-space analyses", Phys. Rev., 48, 17217-17232.

[6] Buot, F. A. and Jensen, K. L. (1991). "Intrinsic highfrequency oscillations and equivalent circuit model in the negative differential resistance region of resonant tunneling devices", Int. J. Comp. Math. Elec. Electron. Eng., COMPEL 10, 241-253.

[7] Woolard, D. L., Buot, F. A., Rhodes, D. L., Lu, X. L., Lux, R. A. and Perlman, B. S. (1996). "On the different roles of hysteresis and intrinsic oscillations in resonant tunneling structures", J. Appl. Phys., 79, 1515-1525.

[8] Sollner, T. C. L. G., Goodhue, W. D., Tannewald, P. E., Parker, C. D. and Peck, D. D. (1983). "Resonant tunneling through quantum wells at frequencies up to 2.5 THz", Appl. Phys. Lett., 43, 588-590.

[9] Buot, F. A. and Rajagopal, A. K. (1994). "Hysteresis of trapped charge in AlGaSb barrier as a mechanism for the current bistability in $\mathrm{AlGaSb} / \mathrm{InAs} / \mathrm{AlGaSb}$ double-barrier structures", Appl. Phys. Lett., 64, 2994-29964; 1994 experimental measurement of $I-V$ was reported by D. H. Chow and J. N. Schulman, "Intrinsic bistability in In As/ $\mathrm{Al}_{x} \mathrm{Ga}_{1-x} \mathrm{Sb}$ resonant tunneling devices", Appl. Phys. Lett., 64, 76-78.

[10] Buot, F. A. and Rajagopal, A. K. (1995). "Theory of novel nonlinear quantum transport effects in resonant tunneling structures", Mat. Sci. Eng., B35, 303-317.

[11] Yoon, H. W. and Pfeiffer, L. N. (1996). "Spatial transport of free trions in mixed-type GaAs/AlAs quantum wells", Bull. Am. Phys. Soc., 41, 239.

[12] See e.g., Paddeu, S., Erokhin, V. and Nicolini, C. (1996). Thin Sol. Films, 284-285, 854.

[13] Pimpale, A., Landsberg, P. T., Bonilla, L. L. and Velarde, M. G. (1981). "Limit cycle in a bound exciton recombina- tion model in non-equilibrium semiconductors", $J$. Phys. Chem. Solids., 42, 873-881.

[14] Nayfeh, A. H. (1981). Introduction to Perturbation Techniques, NY: John Wiley.

[15] Zener, C. (1934). "Non-adiabatic crossing of energy levels", Proc. Roy. Soc. London, A137, 696-702, 1932. See also C. Zener, "A theory of dielectric breakdown of solid dielectrics”, Proc. Roy. Soc. London, A145, 523-529.

\section{Author Biography}

Dr. Felix A. Buot has served on the research staff of the University of London, ICTP (Trieste, Italy), McGill, St. Francis Xavier, Stanford, and Cornell University. He is a Fellow of the Washington Academy of Sciences, and President of the Philippine-American Academy of Science and Engineering. $\mathrm{He}$ is a member of the Editorial Board, Transport Theory and Statistical Physics. He was a UNDP Consultant, 1993 and 1996. His interests include device performance and reliability, optoelectronics, nonequilibrium quantum theory, multiband dynamics, and physics of computation. He is a Research Physicist at the U.S. Naval Research Laboratory. 

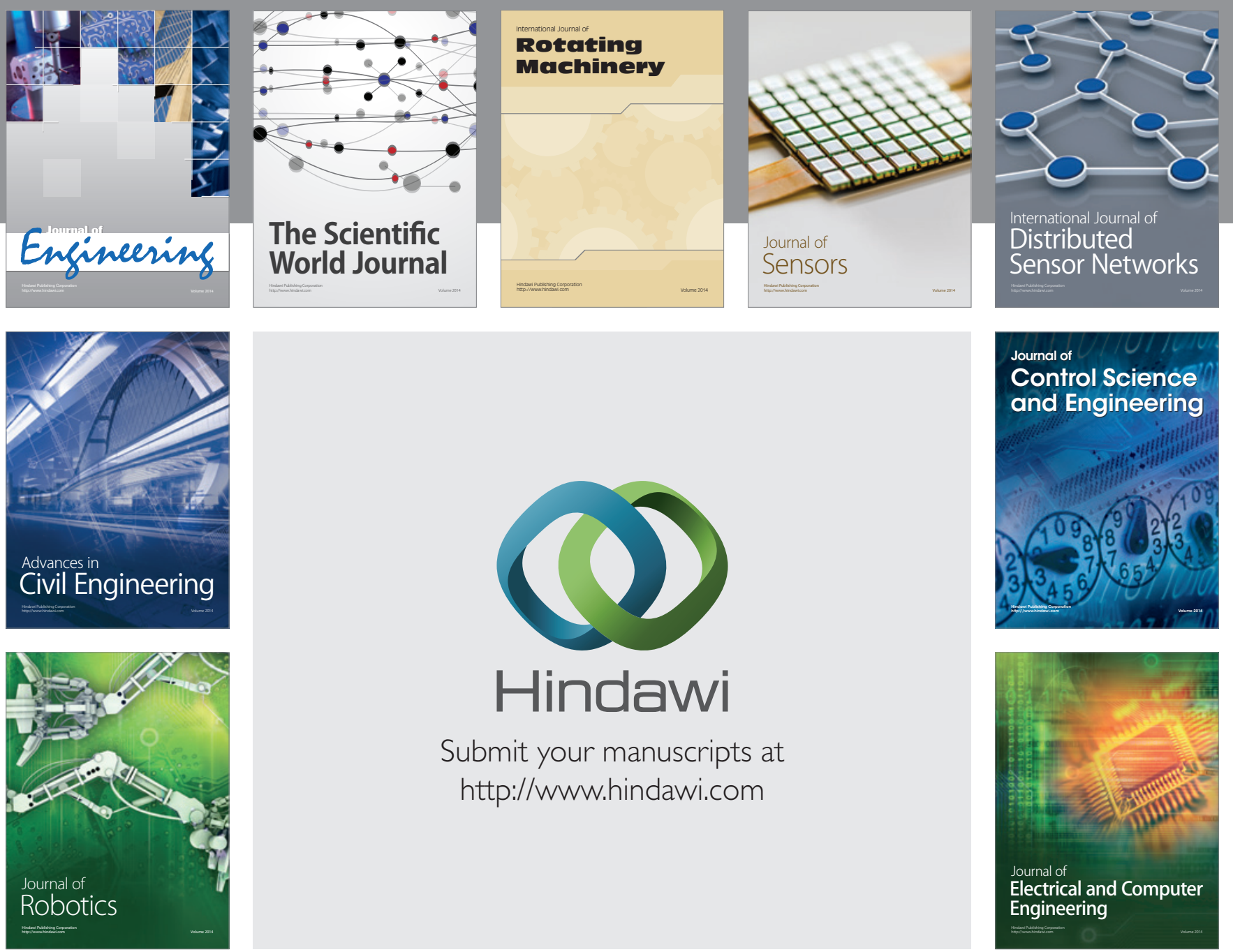

Submit your manuscripts at

http://www.hindawi.com
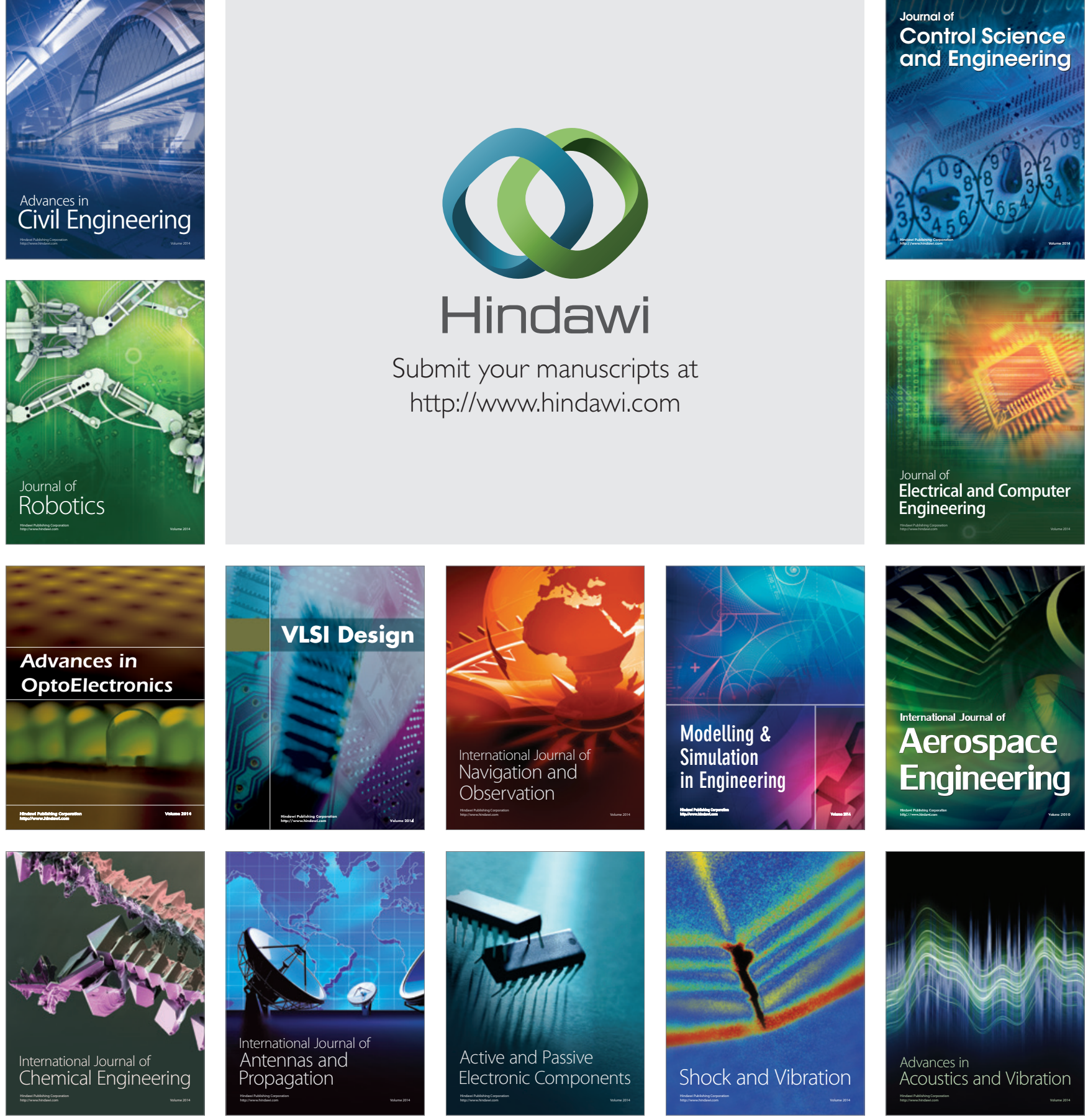